Mering C. STRING v10: Protein-protein interaction networks, integrated over the tree of life. Nucleic Acids Res 2015 January;43:D447-52.

Disclosure of interest None declared

\section{P091 CLUSTERIN IS INCREASED IN EARLY RHEUMATOID ARTHRITIS AND PREDICTS DISEASE ACTIVITY AND TREATMENT RESPONSE}

\author{
1,2T Lennerová* ${ }^{1,2} \mathrm{H}$ Mann, ${ }^{1,2} \mathrm{O}$ Ružicková, 1,20 Šléglová, 'L Vernerová, ${ }^{1,2} \mathrm{~B}$ Šumová, \\ ${ }^{1,2} \mathrm{~K}$ Pavelka, ${ }^{1,2} \mathrm{~J}$ Vencovský, 1,2 L Šenolt. 'Institute of Rheumatology, Prague, Czech \\ Republic; ${ }^{2}$ Department of Rheumatology, 1st Faculty of Medicine, Charles University, \\ Prague, Czech Republic
}

\subsection{6/annrheumdis-2018-EWRR2018.107}

Introduction Clusterin (apolipoprotein J) is a molecular chaperone involved in a number of biological processes, such as inflammation and apoptosis. Recent data suggest its role in the development of autoimmune diseases and bone erosions.

Objectives The aim of this study was to analyse the serum levels of clusterin in patients with early rheumatoid arthritis (RA) and in healthy controls, and to investigate the association of clusterin with disease activity and treatment response.

Methods Clusterin serum levels were measured by ELISA (BioVendor) in 56 patients with early RA before and three months after treatment initiation, and in 56 age-/sex-matched healthy individuals. Disease activity was assessed by 28 -joint Disease Activity Score (DAS28), Simplified Disease Activity Index (SDAI) and Clinical Disease Activity Index (CDAI). Treatment response was calculated based on the SDAI/CDAI definition. Receiver operating characteristic (ROC) curve analysis was performed to predict disease activity and treatment response after six months of therapy. Data are presented as mean \pm SD.

Results Concentrations of clusterin at baseline were significantly higher in patients with early RA compared with healthy controls $(75.1 \pm 12.4$ vs $56.7 \pm 9.7, \quad \mathrm{p}<0.001)$. After three months of therapy, clusterin levels decreased and were comparable to those in healthy subjects $(57.7 \pm 9.7$ vs $56.7 \pm 9.7$, $\mathrm{p}>0.05)$. Although clusterin levels did not correlate with disease activity at baseline, they positively correlated with CDAI and SDAI at month $3(\mathrm{r}=0.294, \mathrm{p}=0.030$ and $\mathrm{r}=0.269$, $\mathrm{p}=0.047$, respectively) and at month 6 after treatment initiation $(r=0.318, p=0.021$ and $r=0.339, p=0.013$, respectively). Using ROC analysis, clusterin baseline levels predicted moderate to high disease activity according to CDAI (AUC $=0.829$ (95\% CI: 0.721 to 0.937$), \mathrm{p}<0.001)$ and SDAI $(\mathrm{AUC}=0.709$ (95\% CI: 0.548 to 0.869 ), $\mathrm{p}=0.019$ ), and major treatment response after 6 months of therapy (AUC $=0.696$ (95\% CI: 0.549 to 0.842$), p=0.015$ for both).

Conclusions We demonstrate here for the first time increased clusterin levels in patients with early rheumatoid arthritis and propose clusterin as a predictive biomarker for assessing disease activity and treatment response.

Acknowledgements Supported by the project of MHCR for conceptual development of research organisation 00023728, research project SVV 260373 and project GAUK No. 534217.

Disclosure of interest None declared

\section{P092 MICROARRAY PATHWAY ANALYSIS COMPARING BARICITINIB AND ADALIMUMAB IN MODERATE TO SEVERE RHEUMATOID ARTHRITIS FROM A PHASE 3 STUDY}

${ }^{1} \mathrm{P}$ Emery, ${ }^{2} \mathrm{PC}$ Taylor, ${ }^{3} \mathrm{ME}$ Weinblatt, ${ }^{4} \mathrm{Y}$ Tanaka, ${ }^{5} \mathrm{EC}$ Keystone, ${ }^{6} \mathrm{ER}$ Dow, ${ }^{6} \mathrm{R}$ Higgs, ${ }^{6} \mathrm{WL}$ Macias, ${ }^{6} \mathrm{G}$ Rocha, ${ }^{6} \mathrm{TP}$ Rooney, ${ }^{6} \mathrm{DE}$ Schlichting, ${ }^{6} \mathrm{SH}$ Zuckerman, ${ }^{7} \mathrm{IB}$ Mclnnes, ${ }^{8} \mathrm{~T}$ Holzkaemper*. 'Leeds Musculoskeletal Biomedical Research Unit, the Leeds Institute of Rheumatic and Musculoskeletal Medicine, Leeds; ${ }^{2}$ Nuffield Department of Orthopaedics, Rheumatology and Musculoskeletal Sciences (NDORMS), University of Oxford, Oxford, UK; ${ }^{3}$ Center for Arthritis and Joint Diseases, Brigham and Women's Hospital, Boston, USA; ${ }^{4}$ First Department of Internal Medicine, School of Medicine, University of Occupational and Environmental Health, Kitakyushu, Japan; ${ }^{5}$ University of Toronto, Toronto, Canada; ${ }^{6}$ Eli Lilly and Company, Indianapolis, USA; ${ }^{7}$ Institute of Infection, Immunity and Inflammation, Glasgow, UK; ${ }^{8}$ Presenting on behalf of Eli Lilly and Company, Indianapolis, USA

\subsection{6/annrheumdis-2018-EWRR2018.108}

Introduction In RA-BEAM (NCT01710358), baricitinib (BARI), an oral selective inhibitor of Janus kinase (JAK) 1 and JAK 2, showed significant improvements in patients (pts) with active RA who had an inadequate response to methotrexate compared to placebo (PBO) or adalimumab (ADA).

Objectives To analyse pathways modulated by BARI compared with ADA (both relative to $\mathrm{PBO}$ ) through 12 wks of treatment.

Methods Pts $(n=1307)$ were randomised $3: 3: 2$ to PBO, BARI $4 \mathrm{mg}$ QD, ADA $40 \mathrm{mg}$ q 2 wks. Total RNA extracted from whole blood drawn at baseline (BL), wk4, and wk12 was analysed using the GeneChip Human Transcriptome Array 2.0 (Affymetrix). Data were analysed using a mixed effects model on a $\log 2$ transformed response.

Results There was little overlap of the immune pathways modulated by both BARI and ADA at wk4 with no significant overlap by wk12. BARI downregulated JAK/Signal Transducer and Activator of Transcription (STAT) signalling pathways, like those induced by IFNs, IL-6, GM-CSF, IL-5, and IL-3. Expression of interferon responsive genes (IRGs) was downregulated by BARI and upregulated by ADA. BARI reduced IRGs by $75 \%$ at wk4 in pts that had high IFN gene expression at BL. ADA modulated complement pathways. Of interest, STAT transcripts were reduced at wk4 by BARI (STAT1, 2, 3, 5A, 5B, 6); by wk12 several STATs (STAT 1, 2, 5A) did not differ from PBO. Additional differences were noted in the number of genes modulated by each treatment. BARI modulated more genes than ADA at wks 4 and 12; BARI resulted in more gene modulation at wk12 than at wk4, whereas ADA gene modulation was similar at wks 4 and 12 . Both the numbers and types of genes modulated by BARI diverged further from $\mathrm{ADA}$ at wk12 than at wk4.

Conclusions Gene expression profiling showed significant differences between BARI and ADA treatments. BARI and ADA modulated JAK/STAT or complement pathways, respectively, and the drugs had opposite effects on interferons, indicating different and possibly complementary mechanisms of action of each targeted therapy.

Acknowledgements Study support:

Eli Lilly and Company and Incyte Corporation. Encore of ACR/ARHP-2017 Annual Scientific Meeting, Nov 4-8, 2016; San Diego, CA, USA.

Disclosure of interest None declared 